\title{
REVIEW OF THE PROSEDS ELECTRODYNAMIC TETHER MISSION DEVELOPMENT
}

\author{
Jason A. Vaughn and Leslie Curtis \\ George C. Marshall Space Flight Center \\ Huntsville, AL 35812 \\ Brian E. Gilchrist \\ University of Michigan \\ Ann Arbor, MI 48109 \\ Sven G. Bilén \\ The Pennsylvania State University \\ 213N Hammond Bldg., University Park, PA 16802 \\ Dr. Enrico C. Lorenzini \\ Smithsonian Astrophysical Observatory \\ Harvard-Smithsonian Center for Astrophysics \\ Cambridge, MA 02138
}

\begin{abstract}
The Propulsive Small Expendable Deployer System (ProSEDS) space experiment was ready to fly as a secondary payload on a Delta-II expendable launch vehicle in late March 2003. Concerns raised by the International Space Station (ISS) after the February 2003 Columbia shuttle accident resulted in the delay of the launch of ProSEDS. Issues associated with both the delayed launch date and a change in starting altitude resulted in the ultimate cancellation of the mission.

ProSEDS was intended to deploy a tether (5-km bare wire plus $10-\mathrm{km}$ non-conducting Dyneema) from a Delta-II second stage to achieve adequate electrodynamic drag thrust that would lower the orbit of the system over days - as opposed to months due to atmospheric drag. The experiment was also designed to utilize the tether-generated current to provide limited spacecraft power.

Considerable effort and testing went into developing the ProSEDS system by a dedicated team. Throughout this effort, important technological issues were identified and addressed and this paper discusses some of the important technical issues and hurdles that had to be addressed to successfully prepare for flight. It is intended that this information will be of use for future tether mission and experiment designers.
\end{abstract}

\section{INTRODUCTION}

ProSEDS was an electrodynamic (ED) tether mission designed to demonstrate electrodynamic thrust as a potential propellantless propulsion technology by deorbiting the $\sim 1100-\mathrm{kg}$ second stage of a Delta-II rocket in about 10 days, which otherwise would have taken on the order of a half a year. The ability to remove satellites and rockets without propellant and with minimal active control of the craft offers a new alternative for mission designers needing to address international requirements for spacecraft to be in orbit no longer than 25 years [NSS 1740.14: "Guidelines and Assessment Procedures for Limiting Orbital Debris," NASA, Aug 1995]. Further, ProSEDS was intended to be the first step leading to the demonstration of the technology for orbit-raising (boost), inclination-change, and drag make-up applications.

\section{ProSEDS MISSION DESCRIPTION}

ProSEDS was to fly as a secondary payload on a DELTA-II Global Positioning System (GPS) satellite launch. After the primary GPS payload and third stage were deployed, the Delta-II second stage was to fire to place ProSEDS in a near circular orbit with an altitude of about $275 \mathrm{~km}$. The Delta II would then begin the ProSEDS mission by turning on the ProSEDS computer to control the payload for the remainder of the mission, after the signal to release the endmass and to deploy the tether came from the Delta II once the correct orientation had been established. After the tether had 
been deployed, ProSEDS would have begun what was expected to be an approximately 1-day mission assuming an initial altitude of $275 \mathrm{~km}$.

ProSEDS consisted of two separate hardware platforms, the Instrument Panel (IP) and the Deployer Side (DS). Both of these platforms were diametrically opposing each other around the Delta-II bellyband. The IP hardware consisted of a 10-A rated hollow cathode plasma contactor, primary battery, secondary battery, Power Distribution Box (PDB), a Langmuir Probe Spacecraft Potential (LPSP) electronics box, Differential Ion Flux Probe with Mass (DIFP/M) electronics box, and telemetry transmitter (ProSEDS was designed to use the existing second-stage antennas). The LPSP and DIFP/M probes were mounted on the Delta-II struts ${ }^{1}$. The DS hardware consisted of an on-board computer called the Data System Electronic Box (DSEB); tether and deployer hardware; both a GPS receiver and antenna; and a student-built endmass called Icarus (provided by the University of Michigan). The deployer hardware included the tether canister, which housed the tether, brake mechanism, and the High Voltage Control and Monitor (HVCM). The HVCM was used to switch the tether in and out of the electrical circuit. The DS hardware closely resembled the design of the old Small Expendable Deployer System (SEDS) ${ }^{2}$ with the exception of the HVCM, which was specifically designed for the ProSEDS mission.

Once on orbit, the ProSEDS operation was to begin with tether deployment and then slowly bring the instruments on-line after tether deployment. Once the entire payload was operating, the primary mission would begin and last about five orbits. These first five orbits on the primary battery were to ensure ProSEDS of at least five orbits of data, which was sufficient to meet all primary objectives established for the experiment. After the five orbits were complete, the extended mission phase was to begin. The extended mission phase operated off of the secondary battery, and during this time ProSEDS was to attempt to regulate the charge of the secondary battery using the current collected by the tether. During normal operation, the system was designed to both open and close the tether circuit to collect background plasma data. This data was required to further the development of models of ED tether propulsion.

During the development of the ProSEDS system, several key design issues had to be addressed and tested to ensure the success of the experiment. Early in the design of the experiment the high voltage design was addressed from a systems perspective. The ProSEDS high voltage system consisted of the tether, the HVCM relay box, load resistor, and secondary battery. The high voltage system had to be designed to survive nearly $-2000 \mathrm{~V}$ in the space environment and the system had to be able to survive high voltage relay opening and closing. Also, the controlled deployment of tether to minimize the libration angle of tether after deployment was a critical task for the success of the mission.

Finally to ensure mission success, the ProSEDS team felt that an end-to-end system test was required to verify the system and drive out any unforeseen problems. However, with a $15-\mathrm{km}$-long tether and several components that required operation in a vacuum $\left(<10^{-6}\right.$ torr), a full system end-to-end test was a tough requirement to meet. In the end, a physical tether deployment during the system test was not possible. However, the system software that sent commands for the deployment was used and verified in the full system test, and several tether deployments were done off-line to demonstrate successful deployment of the tether. The results of the high voltage system design, control law, and testing requirements are described in this paper.

\section{TETHER SYSTEM HIGH VOLTAGE DESIGN}

Crucial to system success was the design of the ProSEDS high voltage system, which controlled and harnessed the energy in this type of tether propulsion system. Certainly, past tether missions such as TSS-1

Kevlar Leader

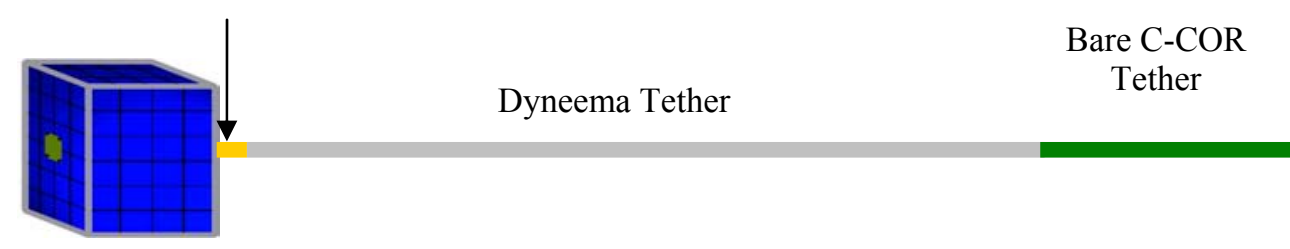

Endmass

Figure 1 ProSEDS Overall Tether Design 
and $-1 \mathrm{R}$ had to meet or exceed similar high voltage requirements. However, the ProSEDS system had the added complication of operating with bare conducting tethers. This type of tether design added complexity and increased the tether design difficulty at least an order or magnitude. For example, because of the bare tether design, electrical connections (i.e., cross straps, which are discussed later) had to be added to connect each electrical wire in the tether together at several points along the length of the tether. These types of design considerations will be addressed in this section.

\section{Tether Triple-Point Design}

The ProSEDS tether, shown in Figure 1, was a $15-\mathrm{km}$ long tether that consisted of a non-conductive ballast tether and a conductive ED tether. The ballast tether was attached to the endmass using a 20-m Kevlar ${ }^{\circledR}$ leader designed to withstand the exhaust plume of the Delta-II motor firing. The Kevlar leader was attached to the $10-\mathrm{km}$ non-conductive Dyneema ${ }^{\circledR}$ section, which was designed with sufficient length to overcome the friction force generated by the ED tether as it exited the deployer canister. The non-conductive tether was attached to the conductive tether using a special Kevlarto-Dyneema splice. This splice was designed to prevent the metallic wire of the conductive tether from coming in contact with the Dyneema due to its low melting point.

The conductive tether has two distinct sections, and each section had unique purposes during the mission. The conductive tether is designed to collect ionospheric electrons on the semi-bare portion of the tether to evaluate the effectiveness of the bare-tether current collection, proposed by Sanmartin et al. ${ }^{3}$ The insulating tether enabled the ProSEDS scientists to open circuit the tether and measure the open-circuit tether voltage. Computer simulations of the 5000-m ED tether, which included late mission dynamics, have predicted the maximum open-circuit tether voltage to be almost $-1400 \mathrm{~V}^{4}$ This prediction was to be verified via on-orbit data.

The conductive tether consisted of a 4800-m semi-bare tether and a $215-\mathrm{m}$ insulated section. The entire conductive tether was made up of seven individually coated 28-AWG aluminum wires. The coating used for the semi-bare tether was an electrically conductive, atomic-oxygen-resistant polymer called ConductiveColorless Oxygen Resistant (C-COR), specifically designed for the ProSEDS mission. The insulating coating consisted of two distinct layers, Triton Oxygen Resistant (TOR) and polyimide. ${ }^{5}$ TOR was an atomicoxygen-resistant polymer that protected the main dielectric layer, the polyimide. Finally, the insulated tether was over-braided with Kevlar for abrasion protection during tether deployment.

An independent high voltage assessment of the entire ProSEDS system was performed early on in the program. ${ }^{6}$ In that assessment the main area of concern for the tether was the triple-point formed when the bare tether, the insulated tether, and the space plasma are collocated. At such locations, electrical breakdowns are common, which can detrimentally affect the tether. The results of space plasma chamber testing on the tether triple point verified that electrical breakdowns with sufficient intensity to sever the tether would occur. ${ }^{7}$ Based on the plasma chamber test results, the tether design was changed to include an application of Aerodag- $G^{\circledR}$ at the transition between the insulated tether and the semi-bare conductive tether. Plasma chamber testing of this transition region demonstrated electrical breakdown, which would occur at $-900 \mathrm{~V}$ without the Aerodag- $\mathrm{G}^{\mathbb{R}}$, now met the $-1400-\mathrm{V}$ design potential with margin ${ }^{7}$.

\section{$\underline{\text { Insulated Tether Pinholes }}$}

Early on in the ProSEDS tether design, the importance of maintaining the integrity of the insulating coating was recognized based on past history with TSS-1R, during which a breach in the insulation led to an electrical discharge event that severed the tether. ${ }^{8}$ During all testing and handling of the flight tethers, every effort was taken to maintain and verify the insulation integrity using a spark test. ${ }^{9}$ Spark tests are a common method used throughout the wire industry for verifying wire insulation integrity. The ProSEDS tether was spark tested using an off the self instrument from Clinton Instruments set at $3000 \mathrm{~V}$ with the center conductor grounded. When a pinhole in the tether passed through the detector an arc occurred shutting down the tether spooling operation at that location. ProSEDS chose the $3000 \mathrm{~V}$ level because it was twice the anticipated tether voltage.

The final flight tethers were processed with one goal in mind: to produce tethers without any pinholes in the 200-m insulated section. During the flight tether processing some pinholes were found and a repair procedure was developed to patch these holes. ${ }^{4}$ Once the final flight tether was completed without pinholes, none of the ground handling, which included simulated tether deployments, produced any pinholes in the insulated tether section.

Pinholes generated by space debris became a concern when ground testing in a plasma chamber indicated a 
small pinhole in the insulation would cause the tether to arc and sever at $-700 \mathrm{~V}$. With predicted tether voltages for the ProSEDS system at $-900 \mathrm{~V}$ in the first few hours, several ground tests were done to verify the test results in a controlled plasma environment. The ProSEDS insulated tether samples, which contained simulated orbital-debris damage experienced plasma discharge between $-700 \mathrm{~V}$ and $-800 \mathrm{~V}$. ${ }^{7}$ Without the needed time to recover from this discovery, combined with the cost of changing the ProSEDS tether design, an operational change was made that eliminated the tether open-circuit mode after the first five orbits. By eliminating the open-circuit mode, the insulated tether would not see high voltage as it always would be grounded to the spacecraft structure. Instead, the high voltage would be shifted to the far end of the tether. At the far end, there was no insulated section and the high voltage would be of positive polarity, which did not show any tendency to arc in plasma chamber testing. ${ }^{7}$

\section{$\underline{\text { Cross Straps }}$}

The ProSEDS conductive tether, particularly the "bare" tether portion, was constructed by twisting seven 28AWG aluminum wires around a Kevlar core. Although the C-COR coating on these wires is moderately conductive, the resistance between adjacent wires is very high (on the order of $\mathrm{M} \Omega$ ). The high resistance between the wires was exacerbated by the twisting design of the tether. Each wire strand was a continuous, end-to-end conductor; hence, if one or more strands were to be broken, either by handling damage or onorbit debris impacts, the current-carrying capability of the entire tether would be reduced. Furthermore, at the location where the wire was broken, there would exist a potential difference between the broken wire and the adjacent, in-tact wires. If this potential were high enough, the broken wire could arc to the other wires, causing further damage to the tether. A third, but less obvious, effect of broken wires is the additional uncertainty this would impose on the understanding and analysis of the electrodynamic performance of the system, since the precise knowledge of the occurrence of broken wires would not be likely. To mitigate the risks posed by broken wires, the idea of electrically connecting the seven wire strands periodically along the tether length was developed. These electrical joints, termed "cross straps", would allow the sharing of the current flow from broken strands to unbroken strands. If these cross straps were applied with enough frequency along the tether, then the overall electrodynamic performance (and end-to-end resistance) of the tether would not suffer, and the potentials near the broken wire ends would be kept low enough the prevent arcing. Thus, the challenge became finding a suitable method for making these cross straps.

The main cross strap requirements were a low resistance connection across the seven wires and that the wire did not overheat while flowing $5 \mathrm{~A}$ of current. The final design involved removing the C-COR coating, wrapping solid copper 34-AWG wire around the tether, and finally overwrapping the copper with Aracon ${ }^{\circledR}$ conductive fibers. Several environmental tests were conducted on the cross strap design including controlled humidity tests, salt water soak, and space environment effects tests. The cross strap did not show any adverse effects to these tests. A typical cross strap is shown in shown in Figure 2.

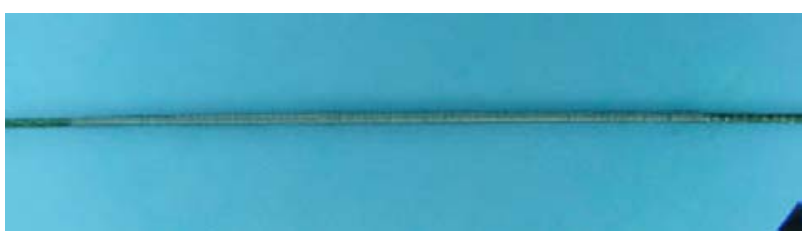

Figure 2 ProSEDS Cross Strap

\section{High Voltage Control \& Monitor (HVCM)}

The primary goal of the High Voltage Control \& Monitor (HVCM) module was to provide control and monitoring functions that supported the validation of the "bare" wire electrodynamic-tether technology in space. Previous electrodynamic-tether flights have used fully insulated tethers with spherical collectors as subsatellites (i.e., TSS-1 and $-1 \mathrm{R}$ ), but collection by a thin, uninsulated wire promises to be more efficient than by a large spherical endmass. Theoretically, the long, uninsulated wire could represent a factor-of-forty increase in electron collection. To properly assess the ProSEDS tether's electrodynamic performance, sufficient knowledge of individual element responses is needed. With the Hollow Cathode Plasma Contactor (HCPC) on, the tether circuit is completed with the ionospheric plasma-the situation to be explored. In order to validate this technology and compare it to theory, several measurements are needed.

In support of these measurements, the HVCM consisted of three high-voltage (HV) relays that switch the tether between open, short (spacecraft ground), resistive load, and battery-charging modes. The HVCM also provided a voltage monitor output, which, when attached to the ProSEDS data system, provided a measurement of the voltage on the tether. The voltage monitor output was protected by an opposed-diode scheme such that, should high voltages temporarily arc across the system, the 
tether voltage should not damage the data system. The load resistor $(\sim 1 \mathrm{k} \Omega)$ was housed in a separate box external to the HVCM module for thermal reasons and was connected to the HVCM via a HV coaxial cable. The output to the secondary battery was diode protected internal to the HVCM to prevent back charging. All $\mathrm{HV}$ inputs/outputs were located opposite of low voltage (LV) input/outputs and the HV was physically isolated from the LV internal to the module. Although the tether current measurement was also a part of the HVCM's responsibility, the ammeter was attached to the ProSEDS deployer system and was not part of the HVCM module.

\section{HVCM Specifics}

A functional circuit diagram of the HVCM is shown in Figure 3. The tether voltage measurement (labeled V1) was designed to measure from 0 to $-1500 \mathrm{~V}$ with a tolerance $<2 \%$ at full scale at a $1-\mathrm{Hz}$ sampling rate. The tether current measured by the ammeter (labeled A1) measured from 0 to $10 \mathrm{~A}$ with a tolerance of $<2 \%$ at full scale at a 1-Hz sampling rate.

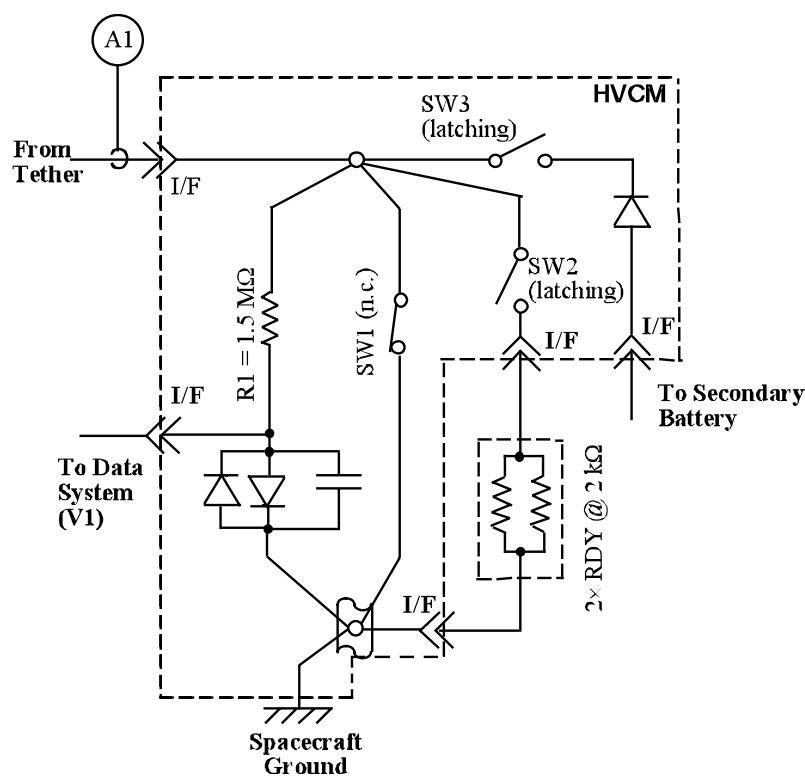

Figure 3: Functional circuit diagram of the High Voltage Control and Monitor (HVCM) for ProSEDS

\section{Tether Current Measurement: Voltmeter-1}

One limitation in estimating the open-circuit emf was due to the presence of some level of tether current flowing in the tether even when it was opened at the lower end via a switch. The presence of the long, bare tether means that the top portion of the tether will be biased positive with respect to the plasma and the lower portion will be biased negative with respect to the plasma. This means that the emf can drive current inducing a voltage drop along the tether, which cannot be measured using a voltmeter on the Delta-II end that accounts for either the voltage drop along the tether nor the positive bias at the upper end. The analysis has shown that an error in emf estimate of 5-7\% exists without compensating for these effects using models. Tether open-circuit currents were estimated to reach $50-100 \mathrm{~mA}$ causing $30-75 \mathrm{~V}$ of total voltage drop. It is also estimated that the "open-circuit" current can cause measurable altitude loss up to $2 \mathrm{~km}$ per day and a measurable change in orbit ellipticity.

The tether voltmeter (V-1) measured the tethergenerated emf with respect to spacecraft (Delta-II) ground. In order for the complete emf to appear across the voltmeter, this measurement must be made during open-circuit (no current flowing) conditions. The maximum emf produced by a $5-\mathrm{km}$ conducting tether was $-1.5 \mathrm{kV}$, but could also have been on the order of a few hundred volts during portions of any given orbit. Therefore, the instrument was designed to measure voltages between 0 and $-1500 \mathrm{~V}$. Overall accuracy requirements were derived from a need for model validation. The open-circuit uncertainty was likely no better than about $\pm 2 \%$ unless a model was utilized to estimate the error. Thus, keeping other errors at a minimum was important to stay close to the expected modeling accuracy. One instrument range should be sufficient and eight-bit measurements will provide $\pm 3 \mathrm{~V}$ resolution over the anticipated voltage range.

The tether voltage monitor circuit consisted of a resistive divider circuit with R1 located in the HVCM relay box and R2 located in the ProSEDS data system. The resistor R2 was located in the feedback loop of an inverting op-amp. A second op-amp inverts and level shifts the measured voltage such that the -1500 to $0 \mathrm{~V}$ from the tether corresponds to 0 to $+5 \mathrm{~V}$, respectively, at the A/D converter. A pair of opposed Schottky diodes were placed between R1 and the op-amps internal to the HVCM relay box to ensure that if a corona should occur across R1 that the tether will be connected to ground through the diodes. In addition, a capacitor was placed between $\mathrm{R} 1$ and the op-amps internal to the HVCM relay box to provide a $0.1 \mu \mathrm{F}$ low-pass filter for the tether voltage measurement.

\section{Tether Current Measurement: Ammeter-1}

The tether current ammeter was a magnetic sensor type (not in-line) capable of measuring currents in the range of 0 to 10 A. Eight-bit data provided $\pm 20 \mathrm{~mA}$ resolution over the anticipated current range. Overall 
accuracy was derived from a need for model validation. For a typical current of $1 \mathrm{~A}, \pm 5 \%$ was well within model accuracy. Therefore the required accuracy should be $\pm 50 \mathrm{~mA}$.

The steady-state current level was not expected to be rapidly varying, hence the measurement frequency can be relatively low; e.g., in the range of $1 \mathrm{~Hz}$. However, recent analysis of the TSS-1R data suggests that current extraction from the ionospheric plasma can be a highly unstable process that oscillates at a high frequency (much greater than $16 \mathrm{~Hz}$ ). Therefore, a measure of $d / d t$ may be important, thus benefiting from higher sampling frequency at times.

\section{HV Relays}

The HVCM controlled the electrical connection of the conducting tether and as such required 3 relays in order to provide the switching functions. Switching occurred approximately once per minute and could occur at any time. The switching sequence was designed in such a manner that all transitions were make-before-break to minimize unavoidable switching transients. In addition, to switching from closed- to open-circuit mode, the load resistor was momentarily connected to dissipate some of the switching transient.

The trade-off made during the selection of relay type was that of power conservation vs. reliability. Latching relays save power, but non-latching versions are likely to be more reliable in terms of mission success. Of the possible failure modes identified, the major concern was the lack of charging current from the tether later in the mission. Since recharging of batteries from the tether had never been done before, there was significant uncertainty. A loss of battery power late in the mission due to inadequate charge would result in the ProSEDS experiment ceasing to have active control of SW1 via its data system. If the batteries are depleted, then a nonlatching, normally closed relay for SW1 would remain closed allowing current to continue to flow through the tether. Other failure modes were possible but have existed in many previous missions and are unlikely. Therefore, we concluded to make SW1 a nonlatching relay.

\section{Load Resistance}

The selection of the load-resistance value was based on the need to validate the electrodynamic-tether models as well as provide information to further refine the models. ProSEDS used two UXP 600 4000- $\Omega$, 600-W resistors in parallel to provide redundancy in case one should open. The UXP 600 resistor is capable of $1000 \mathrm{~W}$ for
10 seconds. The maximum power was calculated using the 208- $\Omega$ tether resistance ("cold" tether at nighttime) along with the 2000- $\Omega$ load resistance and the hypothesis that the maximum voltage and current experienced by the tether while shunted would be 1.5 $\mathrm{kV}$. This calculation yielded $\sim 1500 \mathrm{~W}$. The thermal load of the resistors, which could be conducted into the hardware it is mounted on, necessitated the separation of the resistors from the relay box.

\section{Lessons Learned}

Much of the overall ProSEDS HV design, which includes that of the HVCM itself, was dictated by initial constraints placed on the overall system design. ProSEDS was designed to be a "quick-and-dirty" demonstration system to prove out the bare tether concept. As such, initial system design considerations were to use existing hardware (i.e., the SEDS tether deployer and DSEB) with only minimal modifications to keep mission costs low. Additionally, as a secondary payload, ProSEDS was extremely mass and power limited. Within these constraints, the best design was developed to mitigate against the negative impacts of HV operations, which included electromagnetic interference (EMI), high switching transients, corona, and arcing. ProSEDS lessons learned recommend that the following guidelines be followed in the design of future $\mathrm{HV}$ tether systems.

One of most significant contributions to EMI in the ProSEDS systems was its use of mechanical HV vacuum relays for switching betweens its various modes. This style of relay has a contact time on the order of milliseconds, during which time a fair bit of "bouncing" can occur during closure and contact arcing can occur during opening. This was seen as conducted and radiated EMI, which affect data and power signals. We used the HV vacuum relays due to their heritage on TSS.

The "main" switch that provided an open- or closedcircuit configuration for the tether should be a nonlatching, normally-closed, mechanical relay that would clamp closed upon loss of power in the system. This would allow tethered systems that had failed for a host of reasons (e.g., drained batteries, failed initiation sequence, etc.) to still collect some current along the tether and hence have a chance to decay out of orbit.

Finally, although the design constraints on ProSEDS did not allow for it, the HV section must be physically and electrically isolated from the rest of the payload, preferably via optocouplers. The EMI caused by HV operations and the potential for adverse affects such as 
arcing and corona dictate that there must be no path for these affects to enter the computer and data handling system nor other experiments.

\section{Hollow Cathode}

The hollow cathode plasma contactor (HCPC) for the ProSEDS mission was designed as a small stand-alone system capable of emitting $10 \mathrm{~A}$ of electrons continuously. The HCPC was built by the Electric Propulsion Laboratory (EPL) in Monument, CO using one of their hollow cathodes. ${ }^{10}$ MSFC was responsible for the qualification and acceptance testing of the HCPC. The flight HCPC underwent standard performance testing before and after random vibration testing and before and after shock testing without issue. Figure 4 shows the flight HCPC during final performance testing at MSFC.

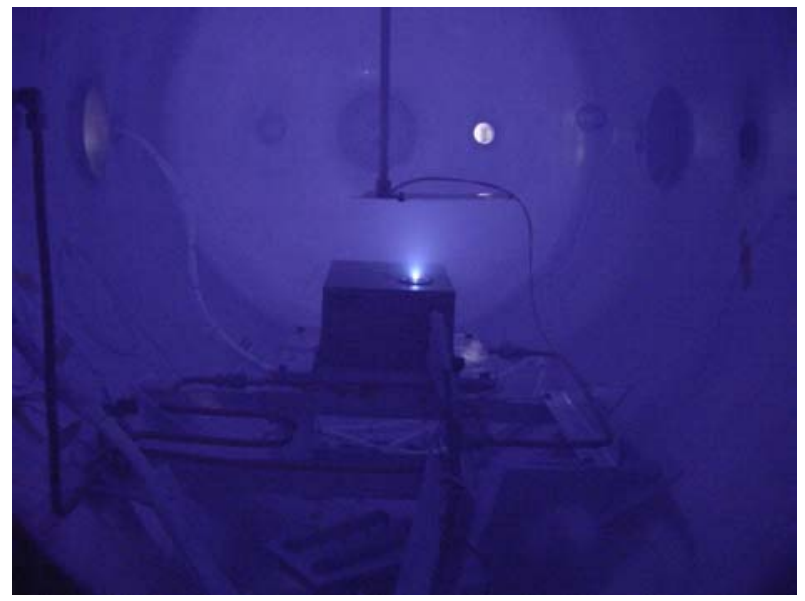

Figure 4 ProSEDS Flight HCPC During Final Performance Testing

The HCPC was subjected to eight thermal cycles that spanned $-12{ }^{\circ} \mathrm{C}$ to $54{ }^{\circ} \mathrm{C}$. At each cold or hot soak point, the HCPC was started and operated for about 30 minutes to demonstrate its cold and hot start limits while on orbit. The HCPC did have some issues trying to start at cold temperature, but the issues were not associated with the Xenon changing phases as might be expected. The primary HCPC cold issue was a single DC to DC converter would not turn on because of excessive leakage current at cold temperatures. However, the HCPC continued to start and operate even with this low temperature anomaly.

Finally, the HCPC was incorporated into the final ProSEDS system test. The objective of the final system test was to test the entire ProSEDS payload in one vacuum chamber for 24 hours. During the system test, the HCPC was able to deliver the electron emission current when required and started on command.

\section{DYNAMICS AND CONTROL}

From the viewpoint of dynamics and control, ProSEDS was built upon the flight success of SEDS-I and II. In fact, high-fidelity flight simulators were already available at the start of the project, such as the Smithsonian Astrophysical Observatory's (SAO) MASTER20 and NASA's GTOSS. These simulators had already been tested on the flight data of the SEDS missions and previously on TSS. Moreover, the deployment control software, developed by SAO, had performed flawlessly during the flight of the SEDS-II mission in 1994. This is a very important point for gaining confidence in a successful project development. There is no way of assessing reliably the accuracy of a space dynamics simulator for a new system unless the results are verified versus flight data of comparable systems. Checking one simulator versus another is no guarantee of reliability of results (and let alone accuracy) unless one of those codes is flight tested. In summary, the foundation for the dynamics and control software to be used for the ProSEDS project had flight heritage.

\section{$\underline{\text { Simulation Tools }}$}

The ProSEDS project required the development of a number of new features to be added to the simulators: (a) a bare-tether collection model in the orbital-motionlimited (OML) collection regime; (b) improving the temperature computation routine of the ED tether to take into account the contribution of tether current and the variation of electrical resistance with temperature; and (c) deployment routines capable of dealing with a tether composed of non-conductive and conductive portions with very different friction characteristics.

These changes were implemented into the SAO simulator MASTER20 then followed by independent changes made by NASA on an improved version of GTOSS. The results of the two simulators were compared for various test cases, modifications were made, and finally two reliable simulation tools were available to the project. The two simulators have been used very extensively, in parallel for some critical cases and separately in many cases to address various aspects of the mission. Examples include the analysis of deployment maneuvers; power profiles produced by the ED tether; dynamic stability of the system; various ionospheric conditions affecting the propulsive performance; the effects of different surface optical 
properties on tether temperature; the coupling between tether dynamics and attitude dynamics of the Delta-II's second stage; the impact of system malfunctions; the effect of orbital altitude on performance; the expected position dispersion in tracking the system from the ground; and other topics not listed here.

Figure 5 shows the ProSEDS expected performance starting from an initial reference altitude of $360 \mathrm{~km}$ as planned for the flight in March 2003. Note that a relatively short ED tether $(5 \mathrm{~km})$ was able to reach $4 \mathrm{~A}$ of current under low density plasma conditions for a launch only a few years away from Solar minimum. In contrast, the 20-km ED tether of TSS-1R reached 1 A of maximum current. This was due to the higher collection efficiency of the bare tether anode as opposed to a large spherical collector. The electrodynamic drag force produced by ProSEDS was estimated to reach 0.5 $\mathrm{N}$ and able to cause a reentry of the Delta-II second stage in slightly over one week's time.

Figure 6 shows details of the system dynamics and the wire temperature (over one day for clarity). The simulation was conservatively initiated with an in-plane libration amplitude of $10^{\circ}$, which was the upper limit requirement for the system. There is interplay in the dynamic response between the in-plane and out-ofplane libration, which is fairly typical of a light ED system with a strong tether current. The tether bowing (measured in the middle of the entire 15-km tether) also shows nonlinear behavior and later grows quickly to a substantial amount stabilizing itself to values of about 3 $\mathrm{km}$. This transition, which was studied in Peleaz et $a l .{ }^{11}$, is growth. The bowing is small during the first two days and is due basically to a nonlinear growth of the tether lateral dynamics forced by electrodynamic forces. These forces pump energy continually into the system and drive a figure-eight-shaped motion of the tether (when viewed from above), the amplitude of which grows non-linearly with time. The growth, however, was not damaging to the propulsive performance of ProSEDS over the decay time thanks to the $10-\mathrm{km}$ stabilization tether. The growth could be mitigated further with active current modulation, which was not an option available to ProSEDS.
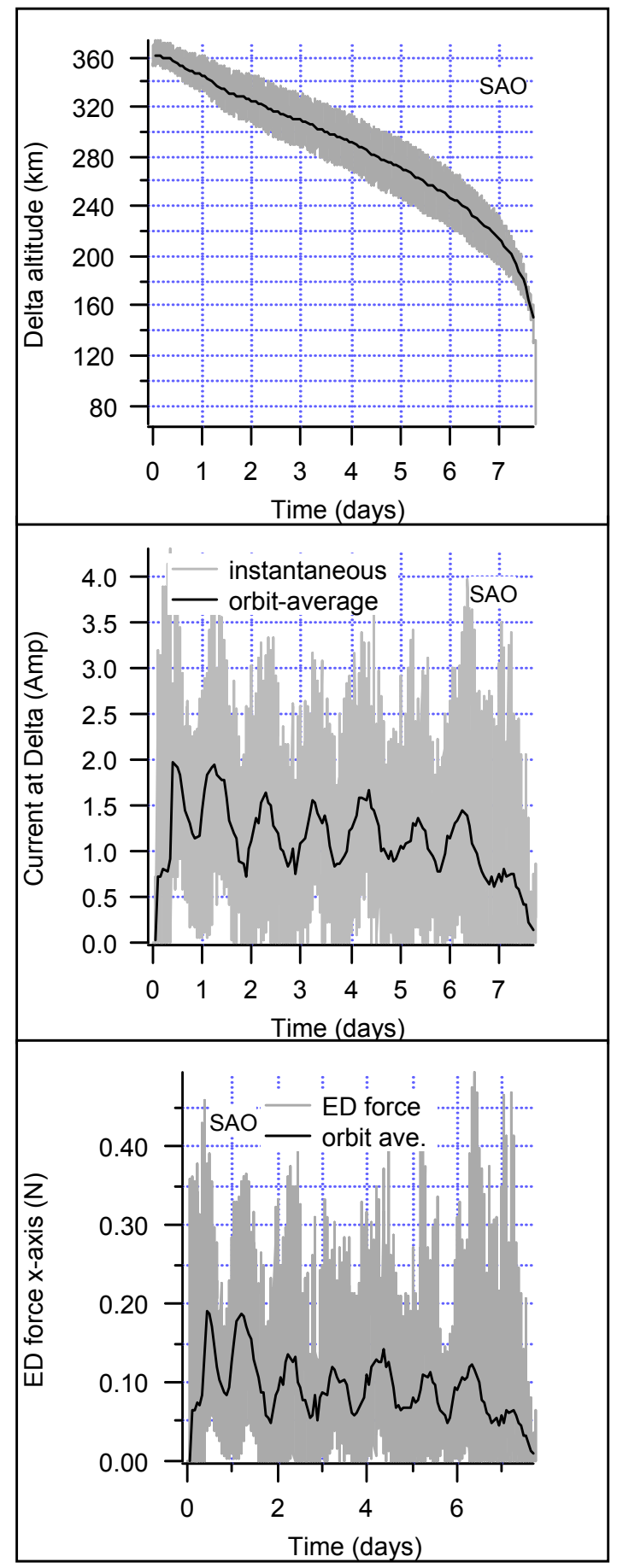

Figure 5 Simulated performance of ProSEDS for a launch in March 2004. 


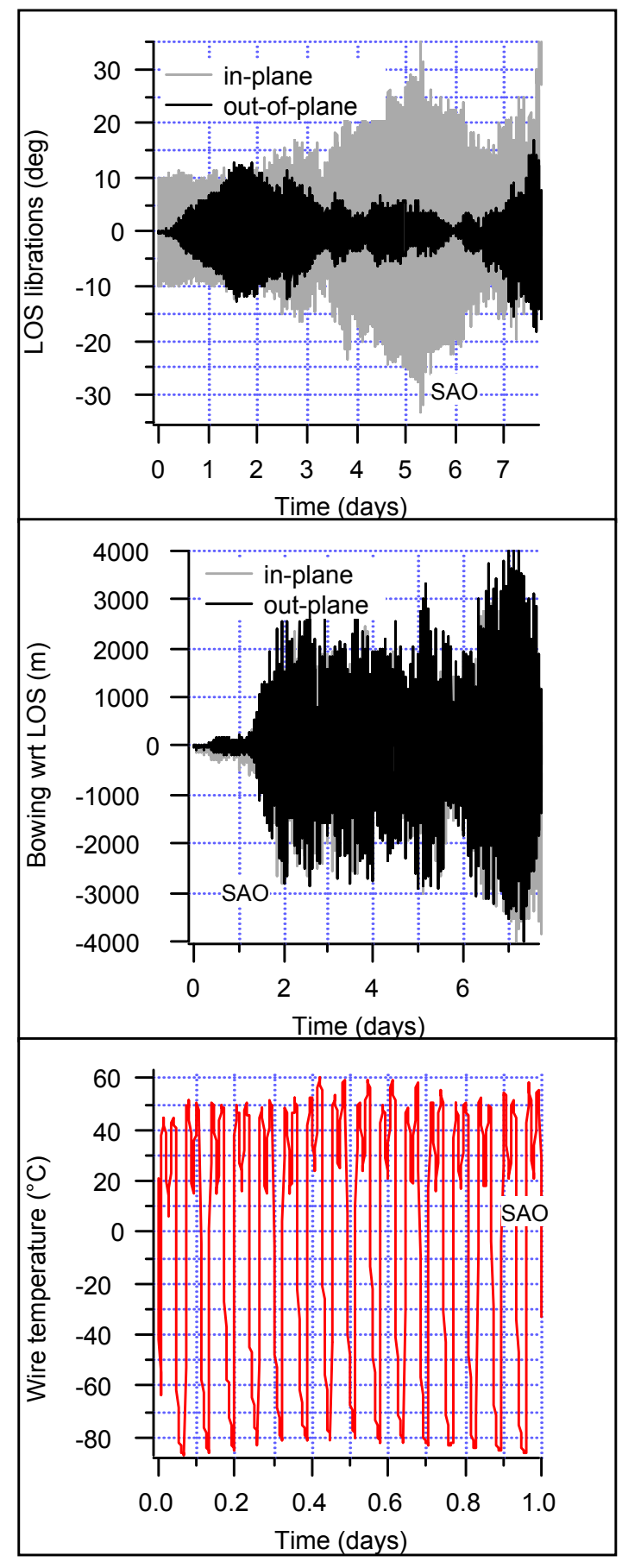

Figure 6 Simulated tether dynamics of ProSEDS and wire temperature.

In summary, reliable simulation tools were critical to the design and development of a new project like ProSEDS. Reliability of simulation tools can only be established through comparison with flight data from similar systems. The ProSEDS project adopted the correct approach in this aspect, thereby reducing the effects of uncertainties on system design and development.

\section{Deployment Strategy}

Deployment of tether systems at sufficiently low speed is a stable maneuver. However, an uncontrolled deployment will leave the system with very large librations at the end of the maneuver. A rapid deployment (i.e., in less than one orbital period) with small final librations is difficult to achieve because the deployment dynamics is non-linear and high tether exit speed produces large libration amplitudes. The difficulties are then compounded by the fact that the forces involved are very small, which implies that hardware and software must act in concert not to stop the deployment prematurely. In fact, with a passive deployment system without any thrusters pushing the tether out, the system can only rely on the initial momentum imparted at ejection and a very feeble gravity gradient.

The SEDS missions demonstrated that passive deployment of a $20-\mathrm{km}$ light tether system can be achieved successfully. Moreover, the flight of SEDS-II proved that deployment could be done in less than an orbit and with a final libration of less than 5 degrees. A major key to the success was the deployment control software that made use of a nonlinear feed-forward, linear feedback control law capable of "eliminating" the nonlinearities and robust with respect to changes in the friction characteristics of the tether/deployer system.

\section{Control law strategy}

ProSEDS utilized the same deployment hardware as SEDS. The deployer, built by Tether Applications, had two successful flights in the 1990s. It is a light deployer of simple and clever design. ${ }^{12}$ However, the actuator (brake) has a highly nonlinear response that makes the design of the control law rather challenging. The available sensors are two photocells that, working together, measure the number of turns deployed from the spool. There are no sensors to measure directly the tether deployment velocity.

The goal of the control law was to control a nonlinear plant, both in terms of dynamics and actuator response, in the presence of uncertainties. The performance goals were similar to SEDS-II: a small final libration amplitude $\left(<10^{\circ}\right.$ desired $)$ and a deployment time of less than one orbit. 
The control law utilized the input-output linearization technique $^{13}$ to eliminate the nonlinearities in both the dynamics and the actuator response. The strategy adopted for deriving the control law divided the control problem into two parts: ${ }^{14}$ (1) the derivation of a nonlinear reference deployment trajectory (in phase space) and the associated brake actuation profile (which are fed forward to the software); and (2) a locally-linear feedback controller that adjusted the brake profile based on the errors between the actual and reference deployment trajectories (in phase space).

The ProSEDS tether, however, was different from the SEDS tether. While SEDS had a uniform $20-\mathrm{km}$ nonconductive tether, ProSEDS had a tether composed of different segments and materials. The ProSEDS tether consisted of a 10-km, non-conductive Dyneema segment (attached to the satellite), followed by a 4.8$\mathrm{km}$ conductive, bare tether and a $215-\mathrm{m}$ insulated conductive tether. Because of its construction, the conductive portion of the tether was not able to tolerate abrasion. Consequently, this tether segment had to be deployed with very small or no braking, thus preventing the use of the closed-loop control law over the entire tether deployment. Finally, the tether exit velocity had to be reduced to a small value during deployment over the last $215-\mathrm{m}$ insulated portion to avoid tension spikes and satellite rebounds.

The SEDS control law (see Lorenzini et al. ${ }^{15}$ ) was adapted to ProSEDS and applied to the deployment of the Dyneema portion of the tether. The reference profile was designed in such a way that feedback control over $10 \mathrm{~km}$ out of the $15 \mathrm{~km}$ was able to limit the final libration amplitude to less than $10^{\circ}$ and for the expected friction variability of the conductive tether. After deployment of the Dyneema portion, the control law was operated in an open-loop fashion. The brake was set to about half a turn during deployment of the wire in order to limit friction and yet reduce the maximum exit velocity. Finally the brake was ramped up over the last $215 \mathrm{~m}$ of insulated tether. This last section was over braided with a Kevlar sleeve that protected it from abrasion. The ramp up was designed as an up-step, flat plateau, and down-step combination that proved to be tolerant (in simulation) of different friction characteristics. The up-step and plateau provided the tether deceleration while the brake's slow removal guaranteed that any tether left came slowly out of the deployer at the end. Figure 7 shows the system response during deployment from pre-flight simulations.

Several deployment tests on the full tether length at different temperatures were conducted at NASAMSFC to support the development of the control software. Tethers have variable friction characteristics that depend on temperature, storage, and other conditions.
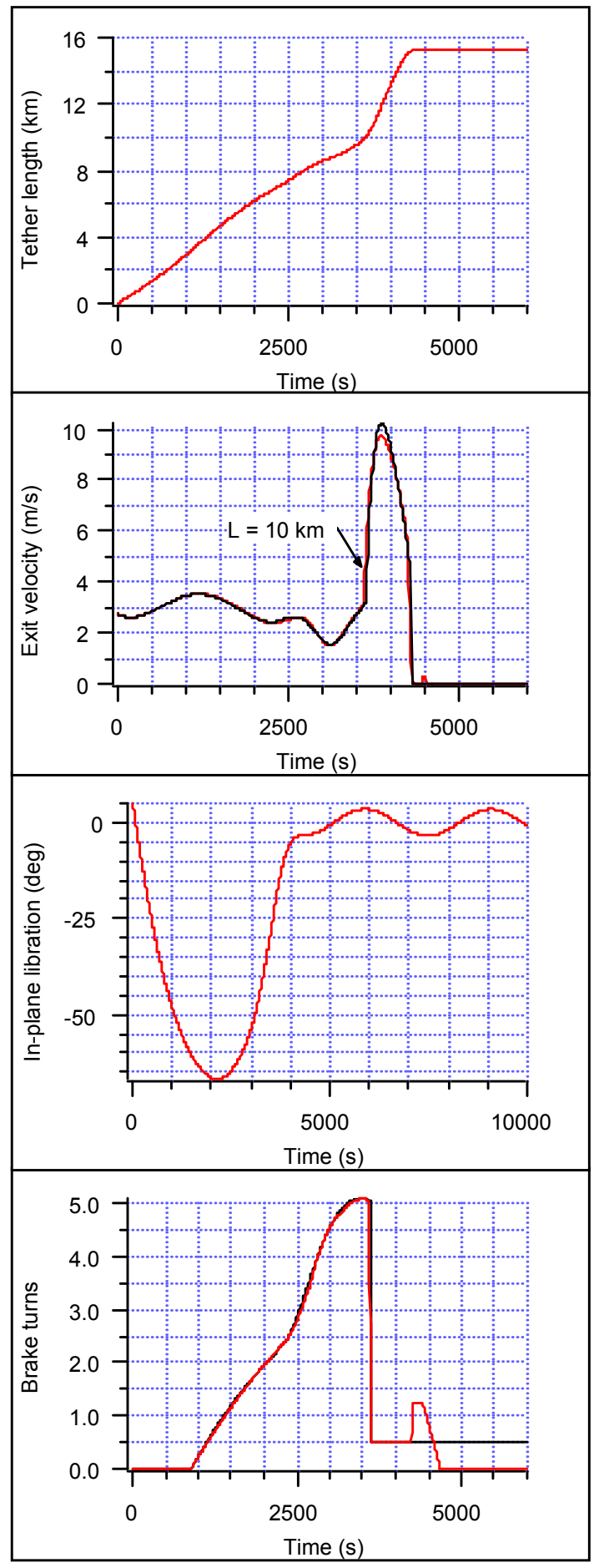

Figure 7 Pre-flight simulation of ProSEDS deployment for typical tether friction. The control law reference profiles are shown in dotted lines. 
Since a passive-deployment scheme is sensitive to friction, it is critical to find out the upper bound of the friction and also the overall range of friction values with which the control law must contend.

The overarching message is that ProSEDS adopted a safe approach to project development. A team with several people who had been involved in multiple flights of similar tethered systems had reliable simulation tools, previously-tested flight control software, and an intimate knowledge of the flight hardware to bring to bear on the development of an incrementally novel system. The development of ProSEDS was nevertheless challenging and required a substantial amount of improvements, fine-tuning, and learning of new lessons. However, the risk involved with the new incremental developments was reduced to a manageable level thanks to the overall knowledge and flight experience of the team.

\section{ProSEDS SYSTEM TEST}

The guidance to "test as you will fly and fly as you test" is impossible to do fully with any ED tether system. There are necessary compromises due to the extreme length of a deployed tether and the difficulty of testing all nuances of HV operations on the ground in a plasma environment within a vacuum chamber. In the systems tests of ProSEDS we employed a "tether circuit simulator" that was developed based on tether circuit modeling by Bilén. ${ }^{16}$ Although only a rough analog of a distributed transmission-line system (i.e., tether surrounded by plasma) in discretized form, the tether simulator we employed was used to stress (but not overstress) ProSEDS during testing. The simulator consisted of 5 lumped circuit elements, each of which included a series resistance and inductance with a parallel capacitance.

ProSEDS was a spacecraft designed and built on a small budget, which eliminated the ability to build prototype hardware for testing. Therefore, to ensure mission success, money was spent testing all components individually and finally testing the system as a whole prior to flight. Because ProSEDS was an experiment with a relatively small number of components, this type of testing was possible.

The ProSEDS system test comprised two different tests. The first test was to combine all flight hardware into the vacuum chamber, monitor the system using external computers, and use a power supply in place of the Primary Battery. This test helped check out the hardware and find any problems associated with combining the flight hardware into one system. In the second test, all external controls were eliminated and the power was provided by the Primary Battery. All data recorded in the second test were received via the transmitter and converted in real time to allow hardware designers to verify the proper operation of each subsystem. Both tests were operated for the 5 orbits of primary battery power through the transition to secondary battery power. However, the first test was completed with ground computer monitoring, and was conducted for the duration of the ProSEDS primary mission, which was 24 hours.

The ProSEDS system test was completed Jan. 2, 2003. For this test, all subsystems were flight hardware with the exception of the tether. Due to physical size limitations, the tether was replaced with a "tether simulator". The tether simulator included a $2-\mathrm{kV}, 5-\mathrm{A}$ power supply, a $250-\Omega$ load resistor, and some small amount of capacitance. Because the tether is a long distributed resistance and capacitance, every effort was made to simulate these distributed elements. However, again size being the limit, the tether simulator contained only five distributed elements.

Figure 8 shows the overall setup for the ProSEDS system test. On the left hand side of the photograph is the deployer side hardware containing the tether canister and the HVCM, on the right hand side at the bottom is instrument panel containing the HCPC and two batteries, in the background is the three DIFP-M probes, and in the fore ground is the stand holding the LPSP probes. As you can see it was quite a challenge to fit all the hardware together but it worked well the way it was configured.

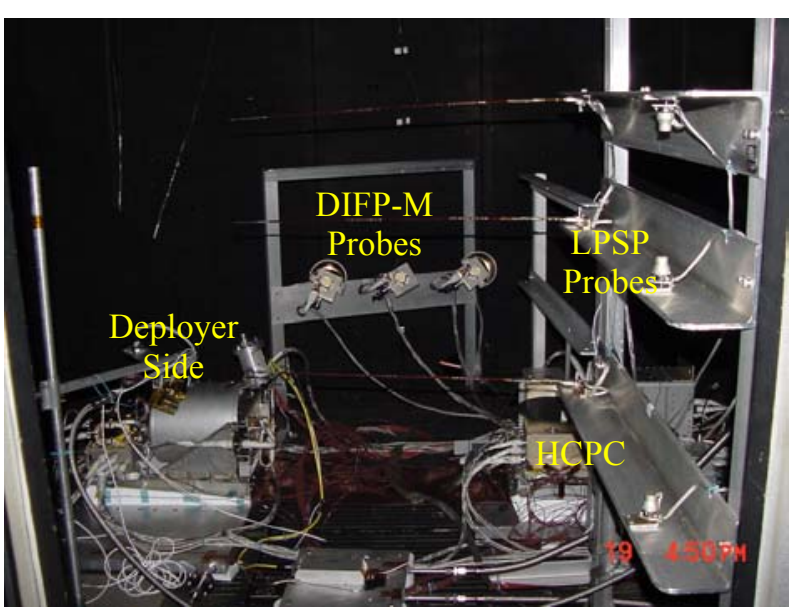

Figure 8 Setup for the ProSEDS system test

\section{SUMMARY}


ProSEDS was a flight experiment designed to demonstrate both the efficiency of the bare tether design and tether technology as a propulsion method. The MSFC-lead ProSEDS team worked hard to overcome many design challenges including overall tether design, the high voltage design, and the control law to prepare for launch. The ProSEDS flight hardware readiness was demonstrated in the final system test that was completed successfully Jan. 2, 2003. Due to circumstances beyond the control of the MSFC team, the project was cancelled. However, this paper documents a number of important lessons learned that will be applicable to the designs of future ED tether systems.

\section{ACKNOWLEDGMENTS}

This research was supported by NASA Marshall Space Flight Center through Grant NAG8-1605 with Leslie Curtis as Technical Monitor.

\section{REFERENCES}

1 Curtis, L., and Johnson, L., "Propulsive Small Expendable Deployer System (ProSEDS)," Space Technology Applications International Forum (STAIF) 2002, February 3-6, 2002.

2 Carroll, J.A., "SEDS Deployer Design and Flight Performance," Proceedings of the 4th International Conference on Tethers in Space, Washington, D.C., April 10, 1995.

3 Sanmartin, J.R., Martinez-Sanchez, M., and Ahedo, E., "Bare Wire Anodes for Electrodynamic Tethers," Journal of Propulsion and Power, Vol. 9, No. 3, pp. 353-360, May-June 1993.

4 Curtis, L., Vaughn, J., Welzyn, K., and Carroll, J., "Development of the Flight Tether for ProSEDS," Space Technology Applications International Forum (STAIF) 2002, February 3-6, 2002.

5 Vaughn, J.A., Finckenor, M.M., Kamenetzky, R.R., Schneider, T., and Schuler, P., "Polymeric Coatings for Electrodynamic Tethers", AIAA 2000-3614, $36^{\text {th }}$ AIAA/ASME/SAE/ASEE Joint Propulsion Conference, Hunstville, AL, July 2000.

6 White, A.B., "ProSEDS High Voltage Assessment," SDL/99-004, Feb. 1, 1999.

7 Vaughn, J.A., Curtis, L., and Welzyn, K., "Plasma Interactions with a Negative Biased Electrodynamic Tether," $8^{\text {th }}$ Spacecraft Charging Conference, Huntsville, AL, Oct. 2003.

8 Szalai, K.J., "TSS-1R Mission Failure Investigation Board, Final Report," May 31, 1996.
9 Clinton, H., "Grounding of Spark Testers," Wire Journal International, March 1996.

10 Aston, G., Aston, M., and Williams, J., "Integrated Hollow Cathodes for Space Applications," AIAA paper 98-3477, 34 ${ }^{\text {th }}$ AIAA/ASME/SAE/ASEE Joint Propulsion Conference, Cleveland, OH, July 13-15, 1998.

11 Pelaez, J., Lorenzini, E.C., Lopez-Rebollal, O., and Ruiz, M., "A New Kind of Instability in Electrodynamic Tethers," Journal of Astronautical Sciences, Vol. 48, No. 4, pp. 449-476, 2000.

12 Carroll, J.A., "Users Guide to SEDS, The Small Expendable-Tether Deployment System," Tether Applications, La Jolla, CA, April 1990.

13 Slotine, J.-J.E. and Li, W., Applied Nonlinear Control, Prentice Hall, 1991.

14 Lorenzini, E.C., Welzyn, K., and Cosmo, M.L., "Expected Deployment Dynamics of ProSEDS," paper AIAA-2003-5095, 39 $9^{\text {th }}$ AIAA/ASME/ SAE/ASEE Joint Propulsion Conference, Huntsville, AL, July 2003.

15 Lorenzini, E.C., Bortolami, S.B., Rupp, C.C., and Angrilli, F., "Control and Flight Performance of Tethered Satellite Small Expendable Deployer System-II," Journal of Guidance, Control and Dynamics, Vol. 19, No. 5, pp. 1148-1156, 1996.

16 Bilén, S.G., Pulse Propagation along Conductors in Low-Density, Cold Plasmas as Applied to Electrodynamic Tethers in the Ionosphere, Ph. D. Thesis, The University of Michigan, 1998. 